\title{
Determination of Bisphenol analogues in infant formula products from India and evaluating the health risk in infants associated with their exposure
}

Kajal Karsauliya ${ }^{1 *}$, Manisha Bhateria ${ }^{1 *}$, Ashish Sonker ${ }^{1,3}$, Sheelendra Pratap Singh ${ }^{1,2,3 \#}$

${ }^{1}$ Pesticide Toxicology Laboratory, Regulatory Toxicology and, Food, Drug \& Chemical Toxicology Group, CSIR-Indian Institute of Toxicology Research (CSIR-IITR), Vishvigyan Bhawan, $31 \quad$ Mahatma Gandhi Marg, Lucknow, 226001, Uttar Pradesh, India

${ }^{2}$ Analytical Chemistry Laboratory, Regulatory Toxicology and, Food, Drug \& Chemical Toxicology Group, CSIR-Indian Institute of Toxicology Research (CSIR-IITR), Vishvigyan Bhawan, 31 Mahatma Gandhi Marg, Lucknow, 226001, Uttar Pradesh, India

${ }^{3}$ Academy of Scientific and Innovative Research (AcSIR), Ghaziabad 201002, India

*Authors contributed equally

\section{\#Address for correspondence}

Dr. Sheelendra Pratap Singh, Senior Scientist

Analytical Chemistry/Pesticide Toxicology Laboratory

CSIR - Indian Institute of Toxicology Research

PO Box 80, Mahatma Gandhi Marg, Lucknow-226001, India

Email: sheelendra@iitr.res.in

Tel: + 91-522-2613786; Ext-667 
Figure S1: Representative MRM chromatograms of Bisphenols in A) (left to right) Acetonitrile Blank, Reagent Blank and Mobile Phase Blank and B) BPA, BPS and BPZ peak detected in samples.

A)

BPA

Acetonitrile Blank

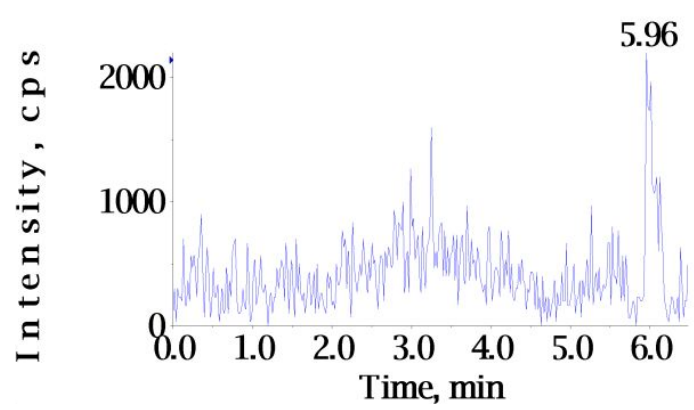

BPAF

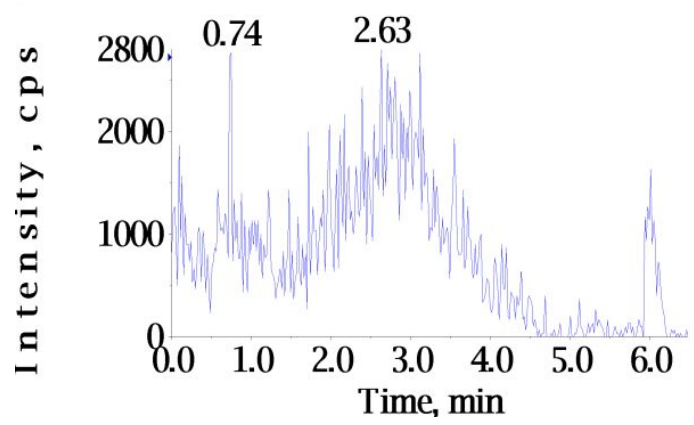

Reagent Blank
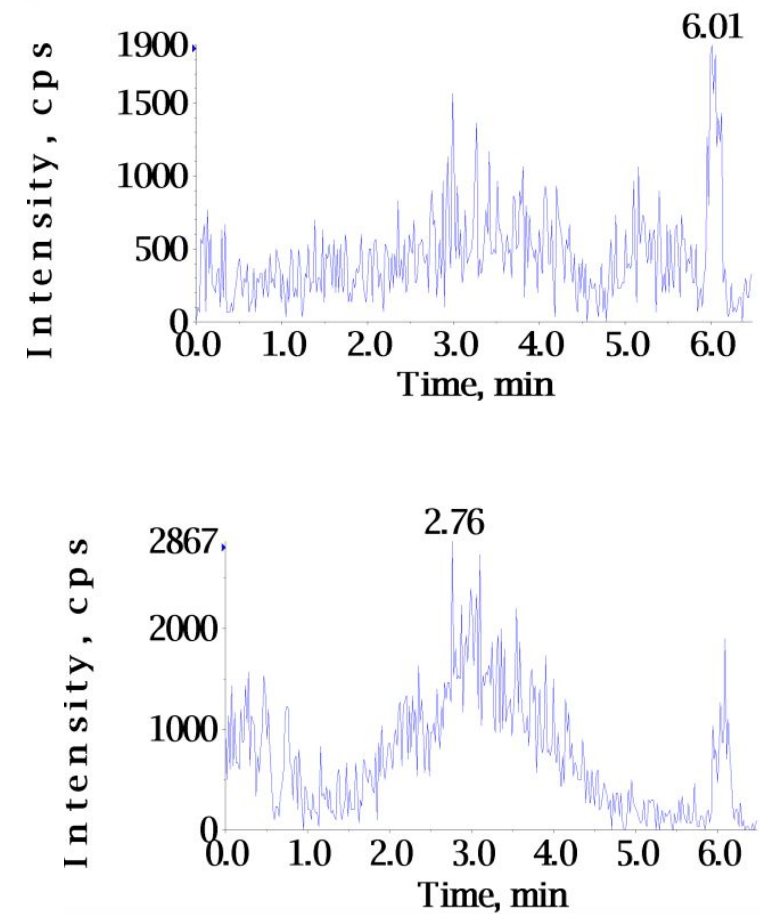

Mobile Phase Blank
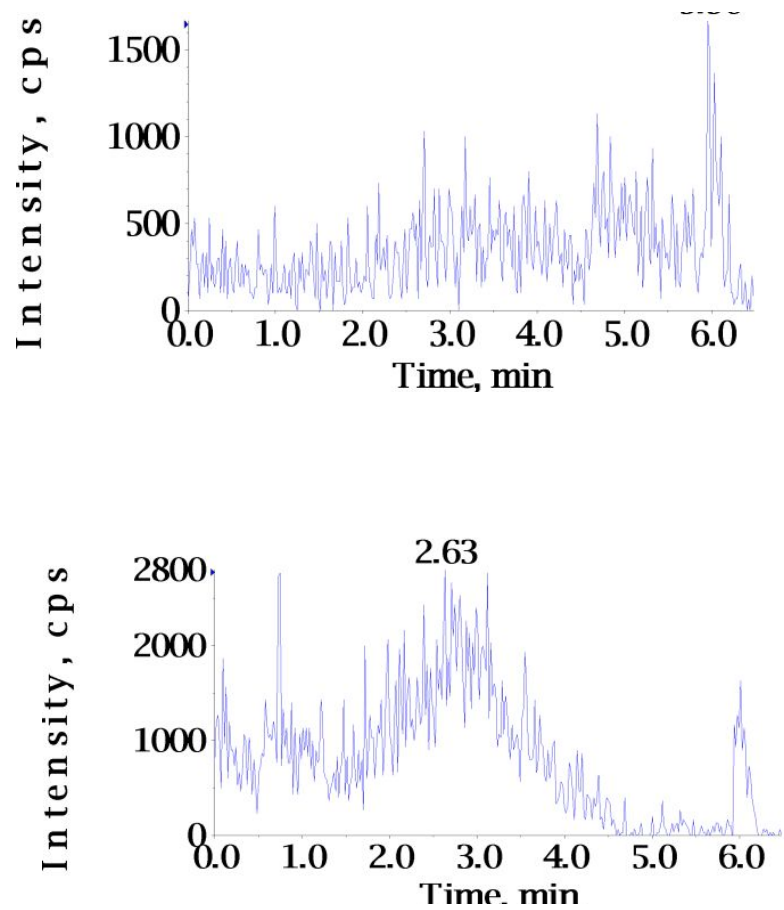

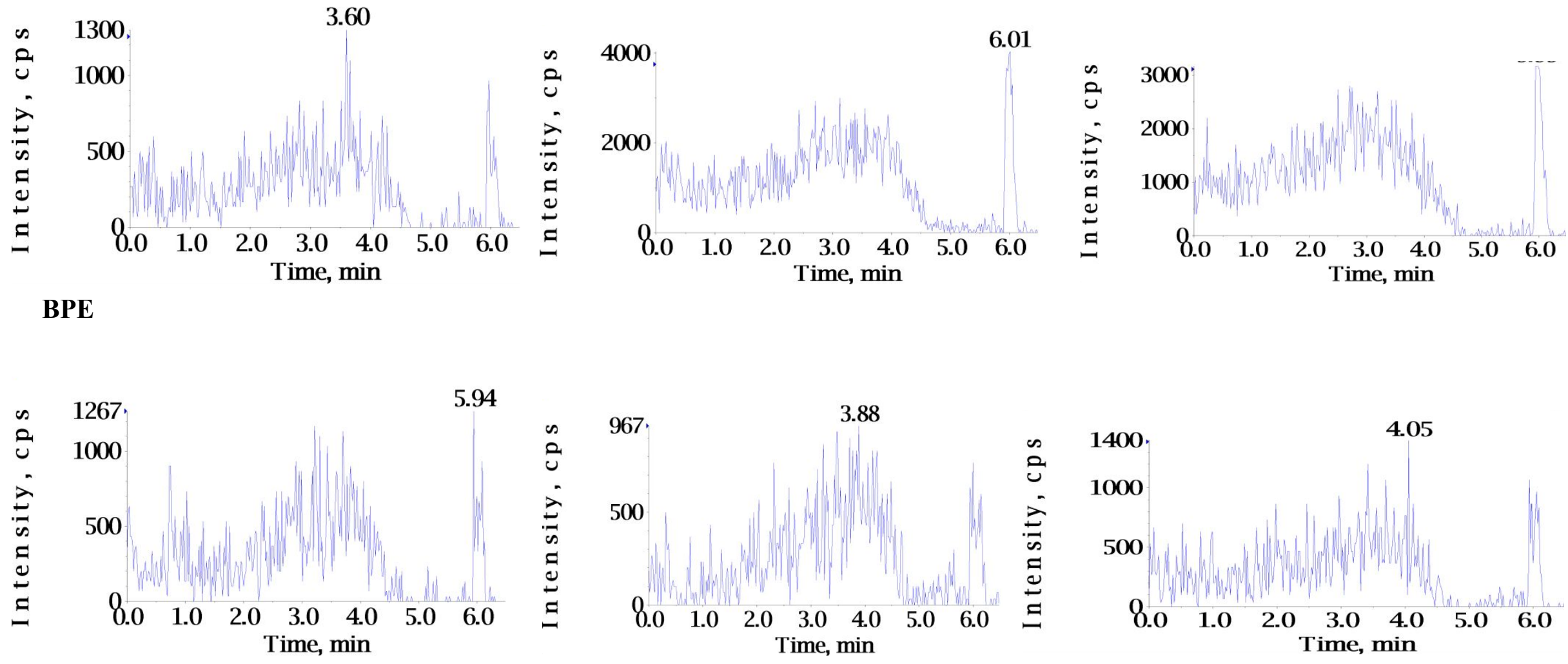

BPFL 

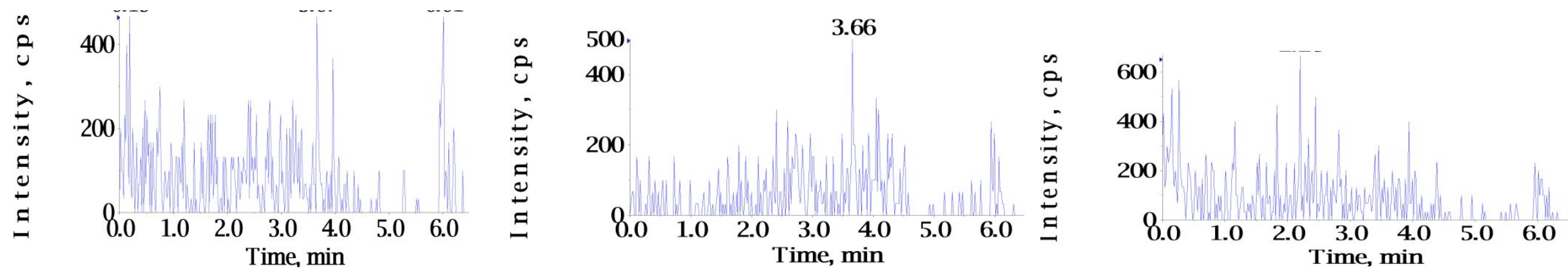

BPS
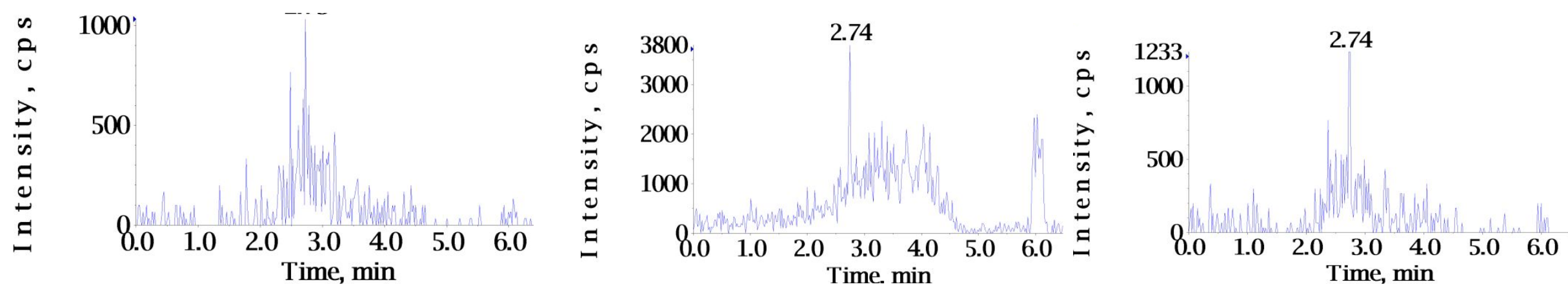

BPZ 


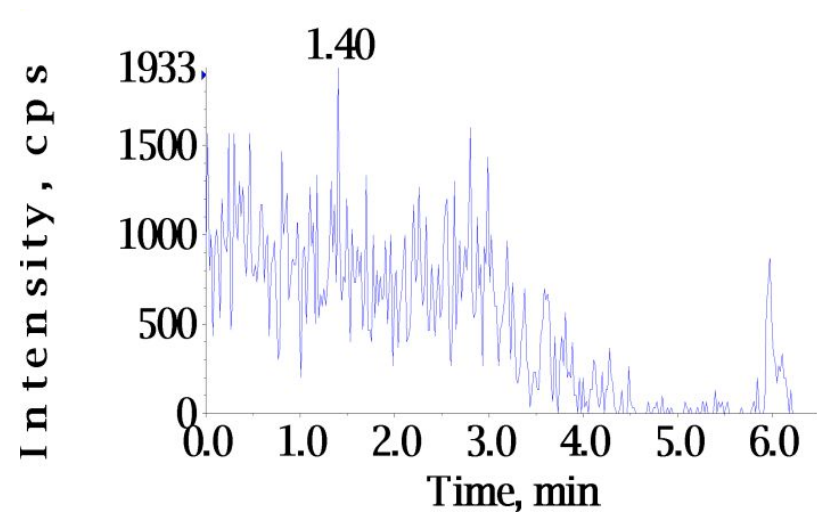

B)

\section{BPA}

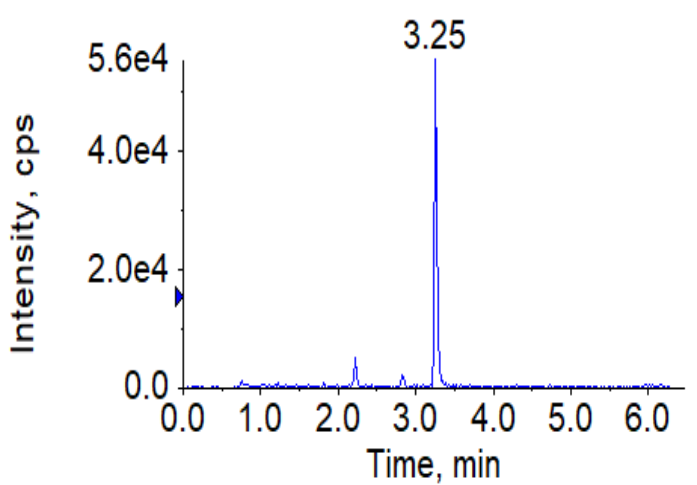

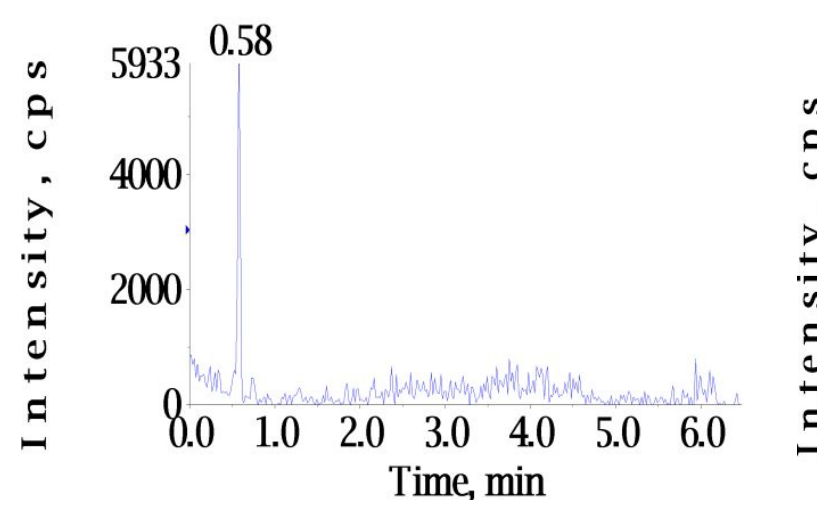

BPS

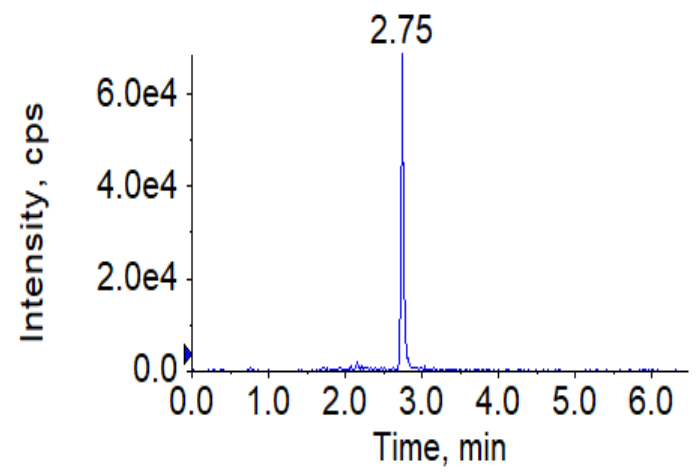

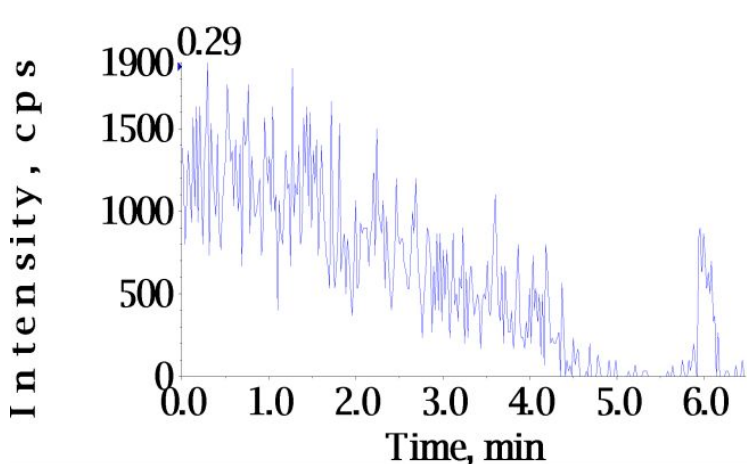

BPZ

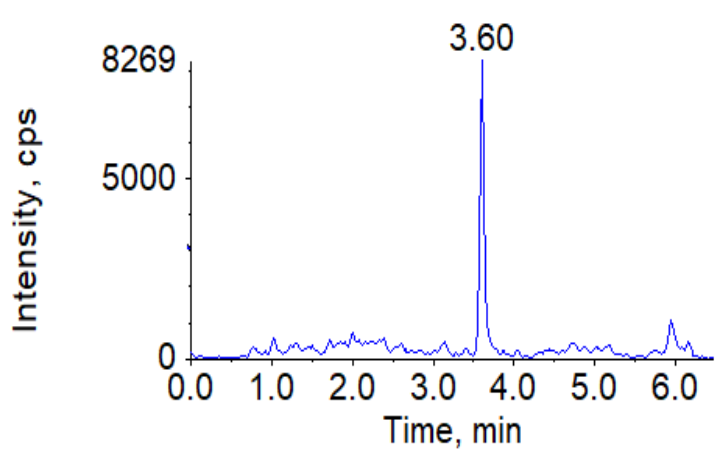


Table S1: The optimization of A) extraction and B) clean-up procedure was done for the simultaneous quantification of bisphenols in infant formula. During the optimization of extraction procedure, the clean-up step consisting of PSA (50 mg) was kept constant; whereas, during the optimization of the clean-up procedure the extraction composition of $\mathrm{NaCl}(1 \mathrm{~g})$, $\mathrm{MgSO}_{4}(2 \mathrm{~g})$ and $\mathrm{ACN}$ was kept constant. The optimization of extraction and clean-up procedure was conducted at single concentration $(10 \mathrm{ng} / \mathrm{mL}, \mathrm{N}=2)$ of bisphenols and the results were evaluated as $\%$ recovery.

\section{A. Optimization of extraction}

\begin{tabular}{|c|c|c|c|c|c|c|c|c|}
\hline \multicolumn{9}{|c|}{ \% Recovery $(\mathrm{N}=2)$} \\
\hline S.No & Variables & $\mathrm{BPA}$ & BPAF & $\mathrm{BPC}$ & BPE & BPFL & BPS & $\mathrm{BPZ}$ \\
\hline 1. & $\begin{array}{c}\text { Extraction: } \\
\mathrm{NaCl} \\
(1 \mathrm{~g})+\mathrm{MgSO} \\
4(2 \mathrm{~g})+\mathrm{ACN} \\
\text { Clean-up: } \\
\text { PSA ( } 50 \\
\text { mg) } \\
\text { Without } \\
\text { sonication }\end{array}$ & $8.94 \pm 0.14$ & $6.88 \pm 0.09$ & $10.59 \pm 0.39$ & $41.13 \pm 0.19$ & $\begin{array}{c}8.22 \pm 0 \\
89\end{array}$ & $\begin{array}{c}13.59 \pm 0.0 \\
8\end{array}$ & $7.59 \pm 0.43$ \\
\hline 2. & $\begin{array}{c}\text { Extraction: } \\
\mathrm{NaCl} \\
(1 \mathrm{~g})+\mathrm{MgSO} \\
4(4 \mathrm{~g})+\mathrm{ACN} \\
\text { Clean-up: } \\
\text { PSA (50 } \\
\text { mg) } \\
\text { Without } \\
\text { sonication }\end{array}$ & $5.83 \pm 0.03$ & $6.37 \pm 0.11$ & $10.04 \pm 0.39$ & $27.50 \pm 0.26$ & $\begin{array}{c}4.78 \pm 0 \\
34\end{array}$ & $\begin{array}{c}14.90 \pm 0.7 \\
0\end{array}$ & $5.38 \pm 0.21$ \\
\hline 3. & $\begin{array}{c}\text { Extraction: } \\
\mathrm{NaCl} \\
(1 \mathrm{~g})+\mathrm{MgSO}\end{array}$ & $4.67 \pm 0.01$ & $\begin{array}{c}13.28 \pm 0.7 \\
6\end{array}$ & $7.37 \pm 0.26$ & $\begin{array}{c}33.55 \pm 0 \\
71\end{array}$ & $\begin{array}{c}3.45 \pm 0 \\
17\end{array}$ & $\begin{array}{c}14.08 \pm 0.5 \\
0\end{array}$ & $5.79 \pm 0.48$ \\
\hline
\end{tabular}




\begin{tabular}{|c|c|c|c|c|c|c|c|c|}
\hline & $\begin{array}{c}4 \\
(2 \mathrm{~g})+\text { Metha } \\
\text { nol } \\
\text { Clean-up: } \\
\text { PSA (50 } \\
\text { mg) } \\
\text { Without } \\
\text { sonication }\end{array}$ & & & & & & & \\
\hline 4. & $\begin{array}{c}\text { Extraction: } \\
\mathrm{NaCl} \\
(1 \mathrm{~g})+\mathrm{MgSO} \\
4(2 \mathrm{~g})+\mathrm{ACN} \\
\text { Clean-up: } \\
\mathrm{PSA}(50 \\
\mathrm{mg}) \\
\text { With } \\
\text { sonication }\end{array}$ & $\begin{array}{c}92.57 \pm 1.1 \\
3\end{array}$ & $\begin{array}{c}92.69 \pm 3.2 \\
6\end{array}$ & $79.65 \pm 1.43$ & $82.37 \pm 3.35$ & $\begin{array}{c}64.68 \pm \\
1.78\end{array}$ & $\begin{array}{c}87.26 \pm 2.5 \\
1\end{array}$ & $92.74 \pm 2.51$ \\
\hline \multicolumn{9}{|c|}{ B. Optimization of clean-up } \\
\hline \multicolumn{9}{|c|}{$\%$ Recovery ( $=2$ ) } \\
\hline S.No & Variables & BPA & BPAF & BPC & BPE & BPFL & BPS & BPZ \\
\hline 1. & $\begin{array}{c}\text { Extraction: } \\
\mathrm{NaCl} \\
(1 \mathrm{~g})+\mathrm{MgSO} \\
4(2 \mathrm{~g})+\mathrm{ACN} \\
\text { Clean-up: } \\
\mathrm{PSA}(20 \\
\mathrm{mg}) \\
\text { With } \\
\text { sonication }\end{array}$ & $\begin{array}{c}91.92 \pm 1.4 \\
4\end{array}$ & $\begin{array}{c}91.66 \pm 0.9 \\
0\end{array}$ & $91.66 \pm 0.90$ & $69.21 \pm 5.58$ & $\begin{array}{c}70.27 \pm \\
0.76\end{array}$ & $\begin{array}{c}93.15 \pm 4.4 \\
5\end{array}$ & $87.10 \pm 2.62$ \\
\hline 2. & $\begin{array}{c}\text { Extraction: } \\
\quad \mathrm{NaCl} \\
(1 \mathrm{~g})+\mathrm{MgSO} \\
{ }_{4}(2 \mathrm{~g})+\mathrm{ACN}\end{array}$ & $\begin{array}{c}95.70 \pm 2.2 \\
7\end{array}$ & $\begin{array}{c}89.28 \pm 1.5 \\
7\end{array}$ & $64.73 \pm 2.47$ & $65.00 \pm 3.35$ & $\begin{array}{l}67.57 \pm \\
1.78\end{array}$ & $\begin{array}{c}88.71 \pm 2.0 \\
5\end{array}$ & $85.02 \pm 2.73$ \\
\hline
\end{tabular}




\begin{tabular}{|c|c|c|c|c|c|c|c|c|}
\hline & $\begin{array}{c}\text { Clean-up: } \\
\text { C18 (5 mg) } \\
\text { With } \\
\text { sonication }\end{array}$ & & & & & & & \\
\hline 3. & $\begin{array}{c}\text { Extraction: } \\
\mathrm{NaCl} \\
(1 \mathrm{~g})+\mathrm{MgSO} \\
4(2 \mathrm{~g})+\mathrm{ACN} \\
\text { Clean-up: } \\
\mathrm{PSA}(50 \\
\text { mg)+C18 (5 } \\
\text { mg) } \\
\text { With } \\
\text { sonication }\end{array}$ & $\begin{array}{c}89.58 \pm 2.6 \\
8\end{array}$ & $\begin{array}{c}92.30 \pm 1.8 \\
0\end{array}$ & $62.71 \pm 2.73$ & $65.79 \pm 2.98$ & $\begin{array}{c}76.22 \pm \\
1.02\end{array}$ & $\begin{array}{c}82.34 \pm 3.9 \\
9\end{array}$ & $81.39 \pm 1.53$ \\
\hline 4. & $\begin{array}{c}\text { Extraction: } \\
\quad \mathrm{NaCl} \\
(1 \mathrm{~g})+\mathrm{MgSO} \\
{ }_{4}(2 \mathrm{~g})+\mathrm{ACN} \\
\text { Clean-up: } \\
\text { Freezing } \\
\text { With } \\
\text { sonication }\end{array}$ & $\begin{array}{c}102.63 \pm 2 . \\
95\end{array}$ & $\begin{array}{c}97.02 \pm 1.9 \\
3\end{array}$ & $91.49 \pm 0.84$ & 86.36 & $\begin{array}{c}94.36 \pm \\
2.32\end{array}$ & $\begin{array}{c}88.65 \pm 3.0 \\
4\end{array}$ & $94.06 \pm 2.32$ \\
\hline
\end{tabular}

